\title{
Intrapulmonary teratoma
}

\author{
M . Y. A L I A N D P.K. WONG \\ From the Departments of Pathology and Medicine, General Hospital, University of Singapore
}

Teratomata arise, in order of frequency, in the ovaries, testes, anterior mediastinum, retroperitoneal region, presacral and coccygeal regions, pineal and other intracranial sites, neck, and abdominal viscera other than the gonads (Willis, 1951). Mediastinal teratomata rank third among all teratoid tumours and are the second most common mediastinal tumours (Inada and Nakano, 1958). The majority of all intrathoracic teratomata are mediastinal, and those occurring in the lungs are among the rarest tumours encountered in pathology (Spencer, 1962).

Before embarking on a review of the literature on intrapulmonary teratomata, it is desirable to establish an acceptable definition of what is meant by a 'teratoma'. Without such a definition, reports of cases designated as 'dermoid cysts' and 'hamartomata' might not lend themselves for classification into this group of tumours. Willis (1951) emphasized the neoplastic nature of the teratoma by defining it as a 'true tumour or neoplasm composed of multiple tissues foreign to the part in which it arises'. This definition excludes pulmonary hamartomata (Liebow, 1952) since no tissues foreign to the lung are present. Dermoid cysts, frequently recorded as synonymous with teratomata (Spencer, 1962), may be included in the group of teratoid tumours provided they contain other tissues besides skin (Willis, 1951).

The following is a brief review of all the cases of intrapulmonary teratomata on record and a report of a new case exhibiting multiple clinicopathological findings.

\section{REVIEW OF THE LITERATURE}

Black and Black (1918) gave a detailed account of their case of pulmonary teratoma which had been surgically resected. That case report, the third in the literature, was the first well recorded case, and the teratoid tumour was found to be malignant. In their discussion they were able to distinguish between genuine teratomata and dermoids on the same criteria as those recently advanced by Willis (1951). The total number of cases reported in the literature since the first one recorded in 1839 are no more than 13 documented cases (Collier, Dowling, Plott and Schneider, 1959; Spencer, 1962; Barrett and Barnard, 1945). The case reported by Barrett and Barnard (1945) is an unusual form of teratoid tumour which showed mixed carcinomatous and sarcomatous elements. Malignant cells were also seen in the sputum before the surgical resection of the lung tumour. Schiødt and Jensen (1960) reported the fourteenth case in the literature. This was a 'sarcomatous teratoid tumour'. Although their histological description does not fully satisfy the criteria for the diagnosis of a teratoma, we have nevertheless included that case as a possibly malignant teratoma. The Table shows a chronological record of all documented intrapulmonary teratomata, including the case under review as the fifteenth case. Six of these were malignant teratoid tumours.

T A B LE

A RECORD OF ALL DOCUMENTED CASE REPORTS OF TERATOMA OF THE LUNG

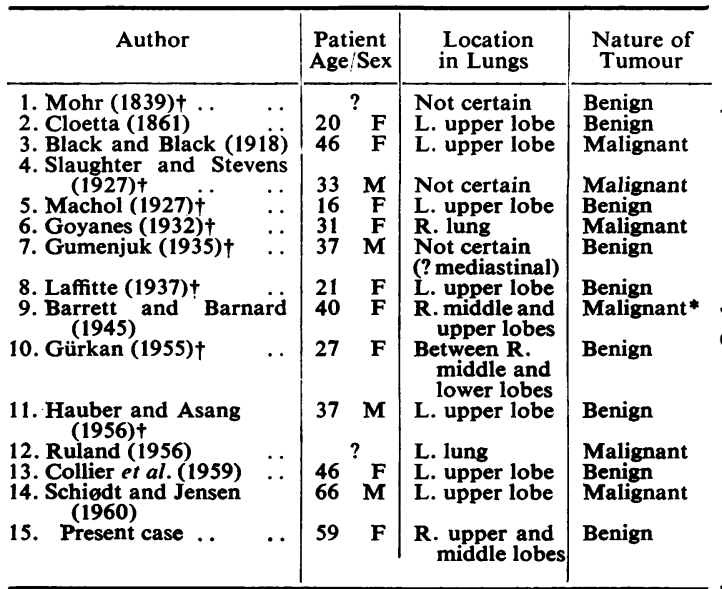

* Patient was well 17 years after the tumour resection (Barrett 1963).

† Cited by Collier et al. (1959). 


\section{CASE REPORT}

C. A.C., a Chinese housewife aged 59 years, was admitted on 10 November 1961 with a history of a week's discomfort in the precordium and upper abdomen accompanied by breathlessness, and a past history of seven years' sporadic cough productive usually of a yellowish sputum. Physical examination showed dyspnoea, moderate cyanosis, and oedema of the lower limbs and over the sacrum. The heart was fibrillating with an apex rate of 80 to 85 per minute, and a pulse rate of about 60 . The apex beat was in the fifth left interspace 4 in. $(101.6 \mathrm{~mm}$.) from the midsternal line. A systolic thrill was palpable over the apex, and a loud rough systolic murmur in the mitral area was heard to be conducted into the axillary region. A short mid-diastolic murmur was also heard in the mitral area. The trachea was slightly shifted to the right. There were some scattered and basal crepitations over the left lung. On the right the percussion note was impaired over the apex and quite dull from the third interspace downwards anteriorly. The breath sounds were bronchial in character over the third to fifth interspace anteriorly. Scattered throughout the right lung were coarse crepitations and rhonchi. The liver was enlarged three finger-breadths and firm. A tentative diagnosis was made of respiratory infection with collapse and consolidation of the right middle lobe complicating mitral valve disease with auricular fibrillation.

The patient was treated with systemic penicillin while investigations were carried out. The haemo-

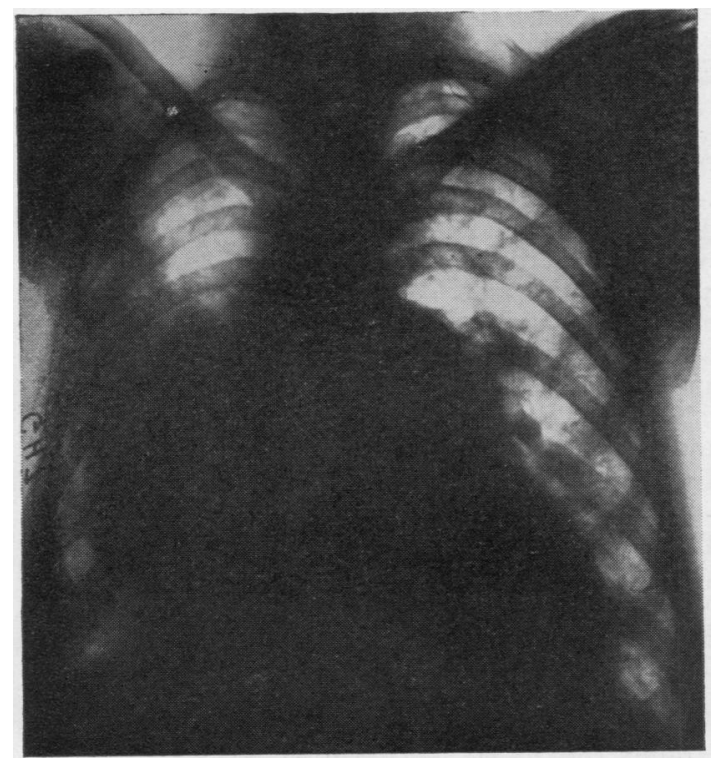

FIG. 1. Chest radiographs: (left) P.A. view showing mass in the right lung; (right) right lateral view. globin was $87 \%$; the white blood cell count was $7,200 /$ c.mm. with a differential count of neutrophils $78 \%$, lymphocytes $15 \%$, eosinophils $3 \%$, and monocytes $4 \%$. The sputum was examined for acid-fast bacilli on numerous occasions (subsequently, more than 40 times) but with no positive results. The sputum was also cultured for acid-fast bacilli and subsequently reported negative on five occasions. A skiagram of the chest showed what appeared to be extensive bronchopneumonic changes with a large shadow in the right middle and lower zones.

A week later signs of respiratory infection had largely cleared up, and the patient was only mildly cyanosed but had signs of collapse and consolidation over the right middle chest anteriorly and a small right pleural effusion. A skiagram showed clearing of most of the shadows, but in the right middle zone the shadows could now be seen to be of a large nonhomogenous rounded mass protruding from the right hilum (Fig. 1).

The electrocardiogram showed right axis deviation and an RSR pattern of right bundle block in the right ventricular leads.

Fluoroscopy showed both right and left ventricular enlargement with marked dilatation of the main pulmonary trunk. The pulmonary arteries in the left upper and middle zones were the only ones visible and were seen to pulsate vigorously. The findings were considered to be consistent with a septal defect. At the same time a cavity was noted in the right middle zone (Fig. 2).

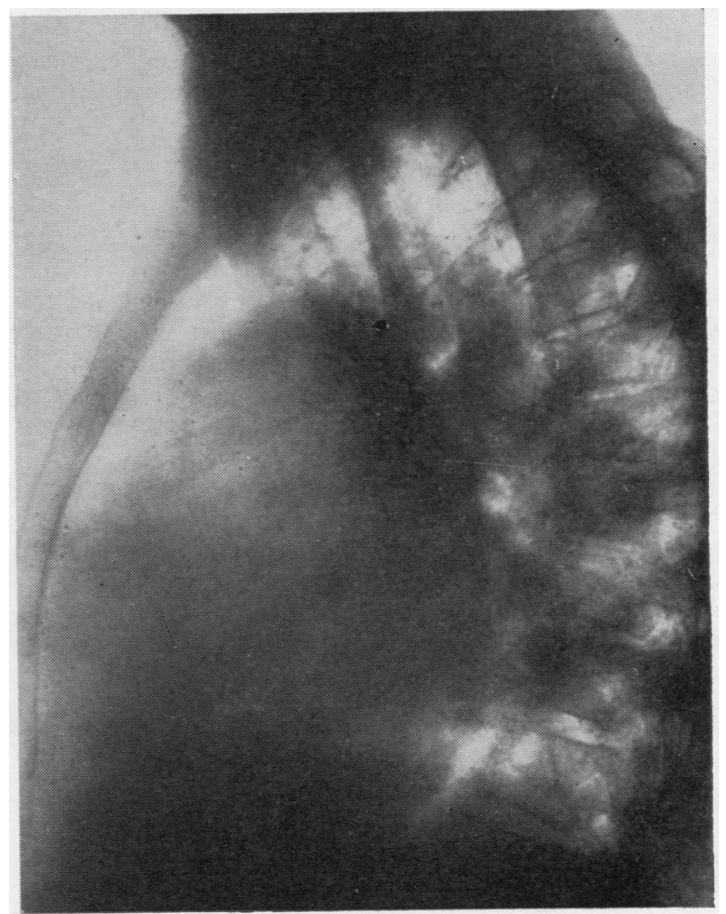




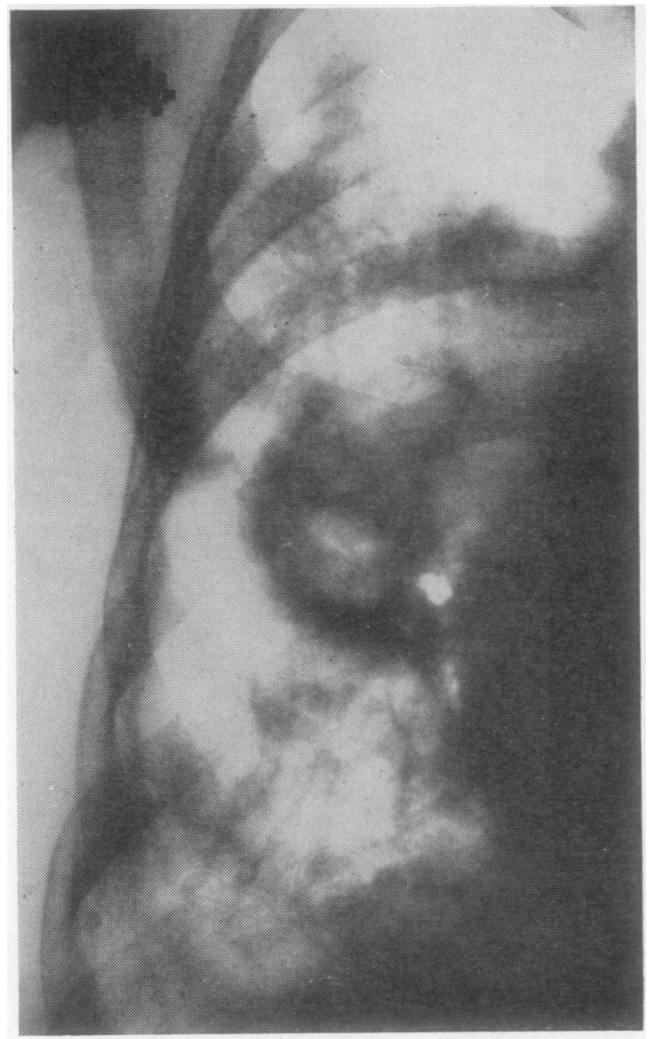

FIG. 2. 'Over-penetrated' chest radiograph showing 'cavity' at periphery of the mass.

Bronchoscopy and bronchography were suggested to the patient, but permission was refused during the next four months while she was followed up as an out-patient, with two admissions for minor complaints. The cardiac signs remained the same except for the disappearance of the mid-diastolic murmur. The auricular fibrillation necessitated the administration of digitalis, $1 \frac{1}{2} \mathrm{gr}$. daily, and the heart rate remained between 80 and 90 per minute. The lung signs remained unchanged.

In March she consented to bronchoscopy and bronchography. The bronchoscope could not be passed beyond the lower trachea, which was narrowed as if by external compression, and a small suspicious elevation on the right side of the lower end was biopsied. This showed no malignant tissue. Bronchography failed.

After this the patient refused further investigations or operative procedures even when in May 1962 she began to have haemoptysis, at times copious enough to require a blood transfusion. Her general condition remained poor, and the heart and lung signs remained unchanged except after episodes of haemoptysis. She eventually died in July 1962 after a few days of haemoptysis at home after which she $\overrightarrow{\overline{\vec{\omega}}}$ was admitted very ill and almost moribund.

NECROPSY FINDINGS (No. 1422/62) The body was that of a pale, wasted Chinese woman of about $60 \mathbb{\Phi}$ years of age. External examination revealed no abnormalities.

Respiratory system The trachea and bronchi con- $\vec{\circ}$ tained frothy, blood-stained mucus. The tracheo-bronchial mucosa was smooth and slightly hyper- $\vec{\omega}$ aemic. The para-bronchial lymph nodes were slightlyo enlarged, firm, and on cut section were pinkish and $\underset{x}{\vec{x}}$ showed no fibrosis.

The right pleural cavity contained about $300 \mathrm{ml} . \overrightarrow{0}$ of clear fluid. The right lung showed fusion of the upper and middle lobes but these were distinctly separated from the lower lobe. The pleural covering $\infty$ of the upper and middle lobes was thickened and slightly nodular in outline on the antero-lateral aspect, $\rightarrow$ but there was no disruption of the pleural covering. The whole of the upper portion of the lung was woody in consistency and airless. On sectioning. the knife $\vec{c}$ met firm fibrous tissue for about $2 \mathrm{~cm}$. thickness sub-o pleurally, the sectioning being directed anteroposteriorly on the long axis. Having cut through the firm subpleural crust, there was a sudden loss ot resistance and a soft area as though cutting through a cyst. The cut surface of the lung (Fig. 3) showedn an easily palpable tumour mass which was incon- $\frac{0}{\mathbb{D}}$ spicuously demarcated from the surrounding lung tissue. The tumour measured 7 by $3.5 \mathrm{~cm}$. and had aó more or less oval shape. The tumour showed a central 'nucleus,' ovoid in shape, measuring 4 by $2 \mathrm{~cm}$. yellowish-white in colour and with a soft smootho consistency. This soft piece of tissue was surrounded by numerous small spherical 'butter-balls' of inspis흐 sated sebaceous material iining a gutter separating iț from the surrounding tumour tissue and only attached to it by a thin stalk (Fig. 3). The surrounding tumous tissue was firm and blended with areas of fibrosis which embraced the tumour. The resultant solid tissue mass impinged anteriorly upon the wall of the righk pulmonary artery and distorted the right main bron윽 chus. The right lower lobe showed areas of fibrosis and marked congestion.

The left lung was uniformly crepitant, congested and oedematous.

Cardiovascular system The aorta showed advancedu atherosclerosis and numerous calcified intimal plaques The pulmonary artery was similarly affected by atheroma. The right pulmonary artery was completely occluded by fresh adherent thrombus (Figs. 3 and 4)을 The heart was large and showed hypertrophy and dila tation of the right ventricle and dilatation of the righ? atrium. There was a gaping atrial septal defect measur $\square$ ing $2.5 \mathrm{~cm}$. in diameter. All the valves and the endo cardium showed no abnormalities.

All other organs Apart from vascular congestion of the liver and spleen, all other organs were essentially normal. The ovaries were fibrotic, consistent wite menopausal change, and showed no neoplasia. 

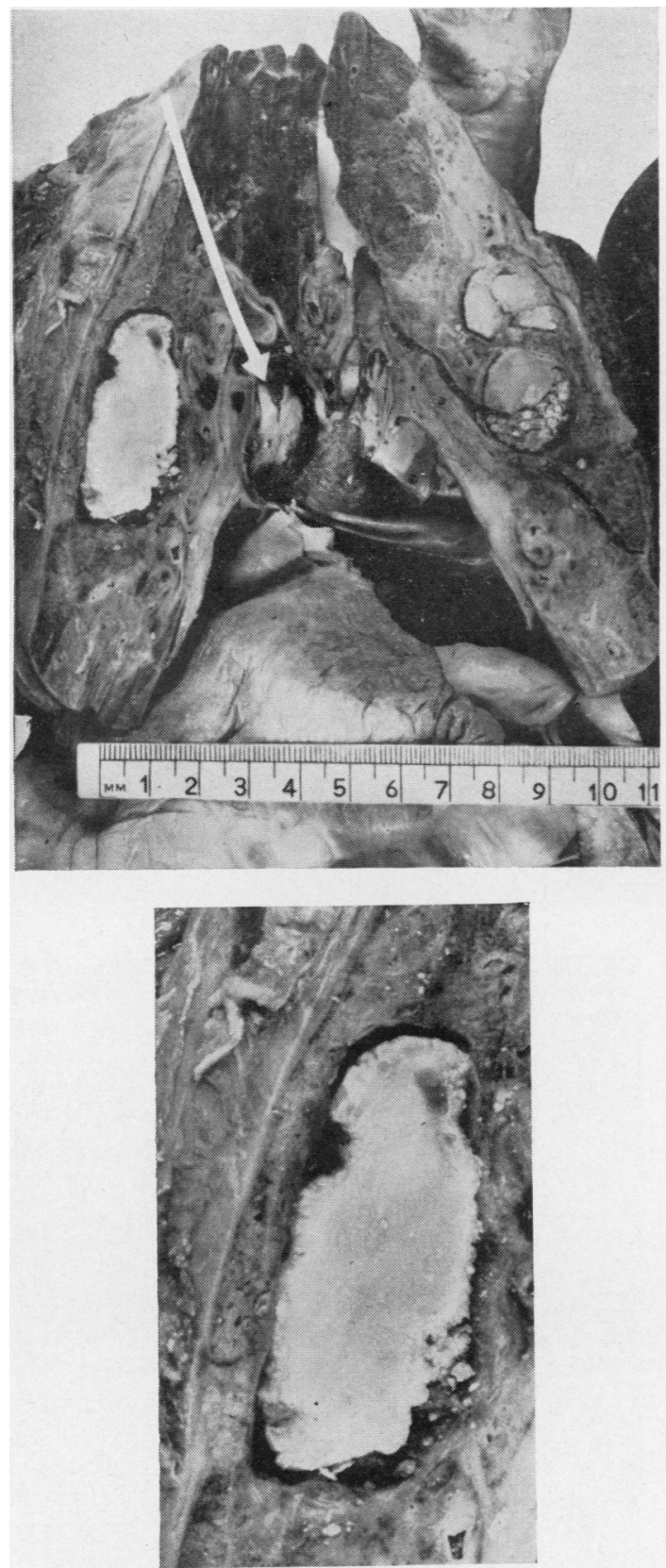

FIG. 3. (above) Gross appearance of a longitudinal section of the right lung showing the 'nucleus' of the tumour and the poorly defined margins of the rest of the teratoma. The arrow points at the thrombosed right pulmonary artery. Note the thickened pleural covering anteriorly. (below) Actual size of the adipose tissue 'nucleus'. Note the appearance of the surrounding sebactous material.

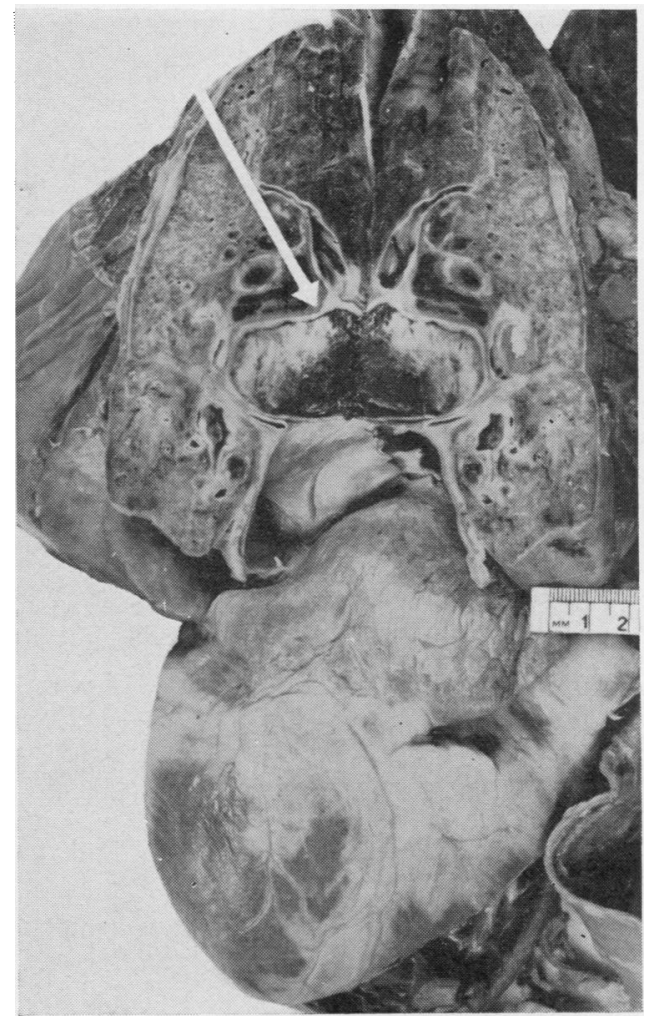

FIG. 4. Longitudinal cut surface of the right lung showing the thrombosed pulmonary artery, indicated by arrow. Note the widespread fibrosis of the lung. The anterior surface of the lung, displaced to the left, shows the thickened pleural surface.

Histopathological examination Numerous sections were collected from various areas of both lungs and the tumour in the right lung. Paraffin sections stained with haematoxylin and eosin was the method used for studying all the lung tissues and also those routinely collected from all other organs.

Sections of the ovoid 'nucleus' of the lung tumour showed adipose tissue, blood vessels, and sebaceous glands clothed by fully developed skin and surrounded by sebaceous material. Sections taken from the surrounding tumour mass showed fully developed skin lining the space between the 'nucleus' and the rest of the tumour. The skin component in this side of the tumour had fully developed appendages, including hair and sebaceous and sweat glands. Scattered among the assembly of various tissues, other than skin and its appendages, were bundles of nervous tissue (Fig. 5 a-d). Apart from those ectodermal elements, osteoid and well-developed pancreatic tissues were seen representing mesodermal and entodermal components scattered without apparent arrangement and, in places, 

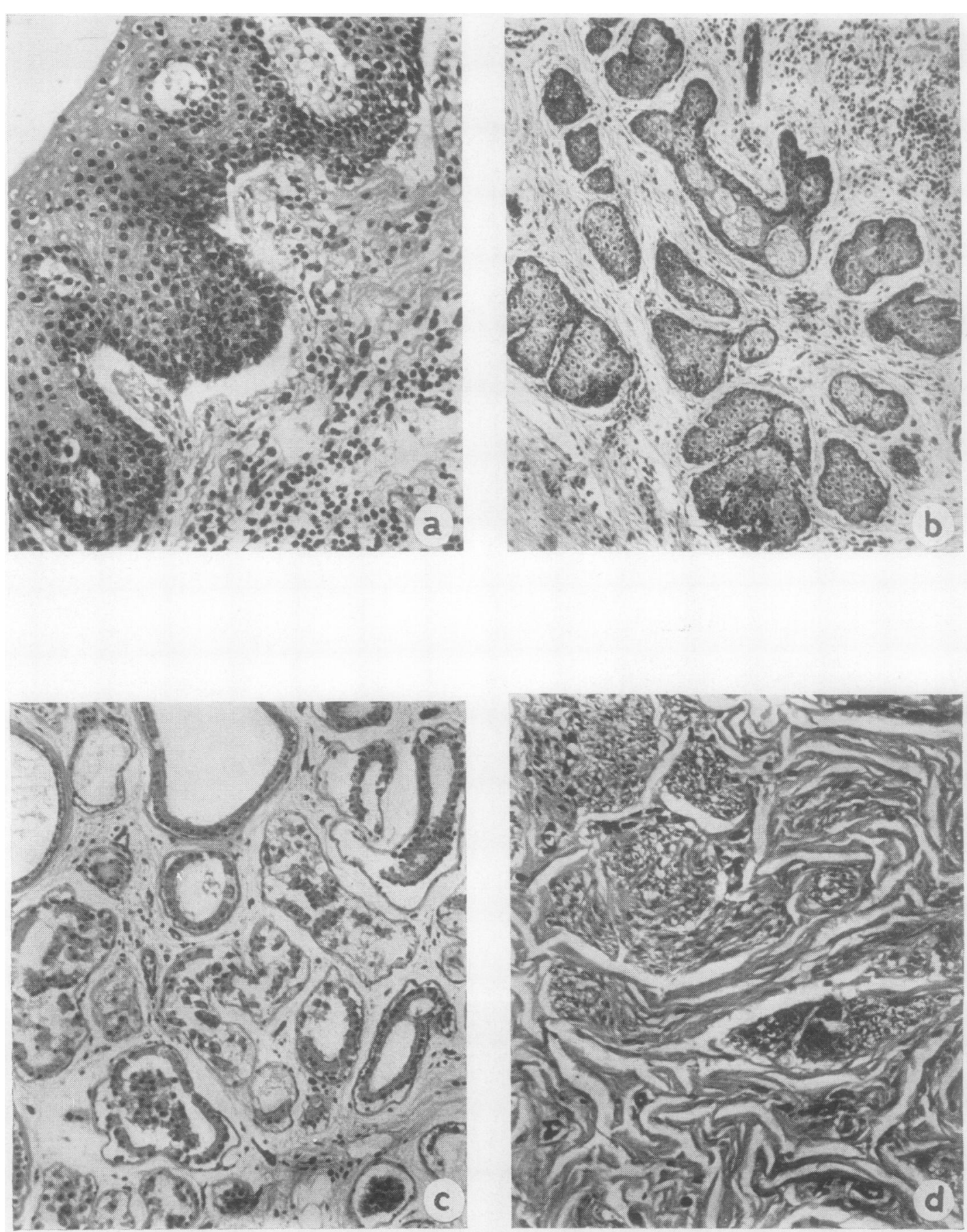

FIG. 5. Ectodermal derivatives of the teratoma: (a) Well-developed skin showing papillae and appendages; (b) large $\stackrel{\mathbb{D}}{\circ}$ masses of sebaceous glands; $(c)$ eccrine and apocrine types of sweat glands; and $(d)$ nerve bundles. Haematoxylin and $\underset{2}{\sigma}$ eosin stain, green filter. $(a)$ and $(d) \times 150 ;(b)$ and $(c) \times 75$. 

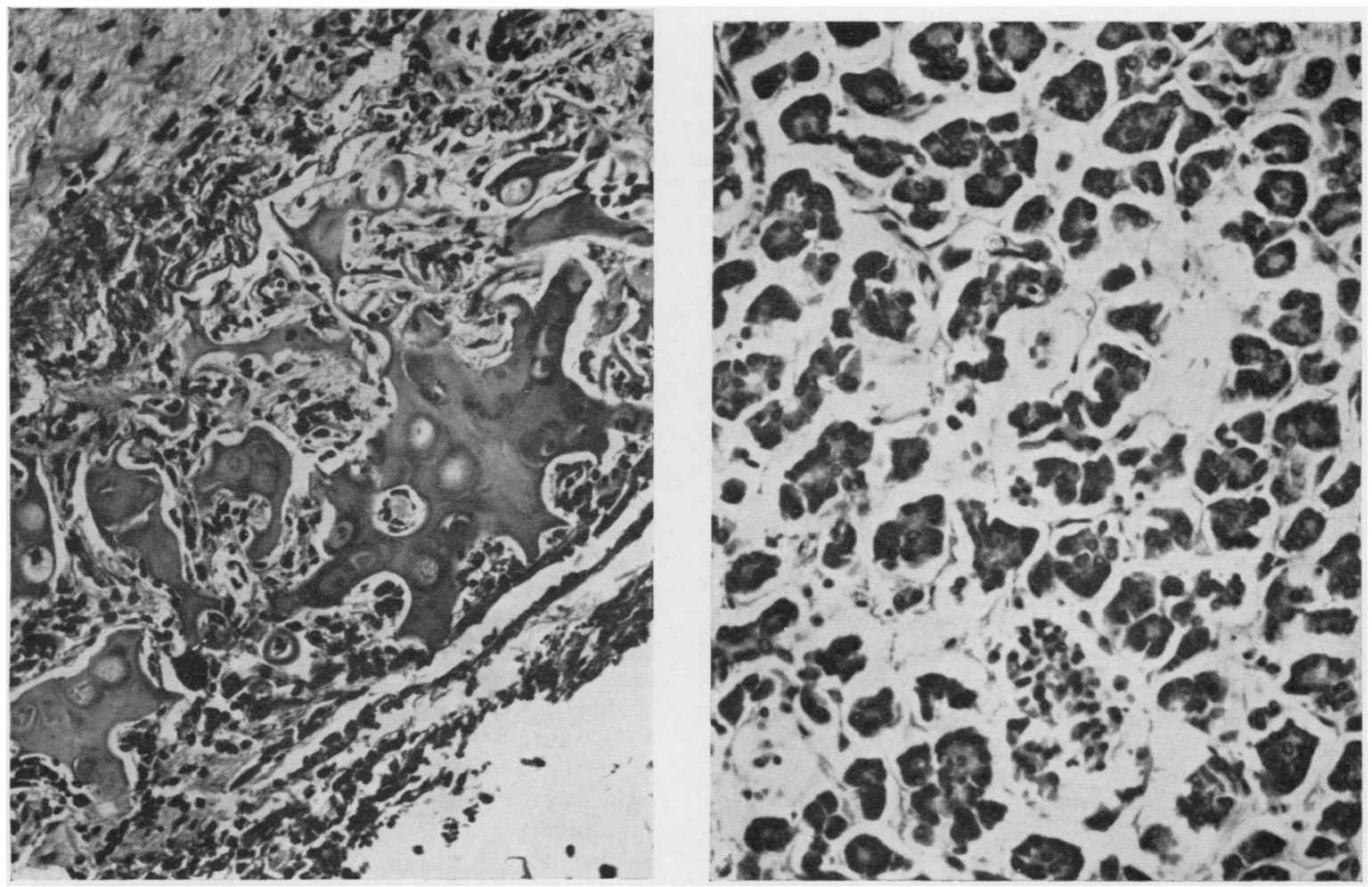

FIG. 6. Mesodermal and entodermal derivatives: (left) osteoid tissue representing mesodermal elements; and (right) pancreas, representing entodermal elements. The islet of Langerhans is seen in the lower half of the field. Haematoxylin and eosin stain, green filter (left) $\times 150 ;($ right $) \times 200$.

seen to merge with the surrounding fibrotic lung tissue (Fig. 6). Large numbers of sections from various areas of the tumour showed no evidence of malignant change in any of the tissue components.

Lung tissues, other than those from the tumour area, showed fibrosis. Grossly crepitant portions of both lungs showed histological evidence of pulmonary hypertension. The para-bronchial lymph nodes showed chronic inflammation and sinus reaction.

Routine sections of all other organs showed no relevant pathological findings.

The necropsy findings may be summarized as follows: (1) Intrapulmonary teratoma in the upper and middle lobes of the right lung ; (2) thrombosis of the right pulmonary artery ; (3) atrial septal defect; and (4) pulmonary hypertension.

\section{DISCUSSION}

A complicating feature in this patient's illness is the presence of the cardiac lesion. It is tempting to link the two congenital anomalies of atrial septal defect and teratoma together, but there is no evidence to suggest a direct link.
The terminal pulmonary artery thrombosis is a feature secondary to pulmonary hypertension that may occur in these patients with atrial septal defect. The earliest episode of dyspnoea and cyanosis must be accepted as respiratory infection although multiple pulmonary infarcts were considered at that time. However, there was no evidence of healed infarcts in the lungs at necropsy.

Clinically, the cardiac lesion first led to the theory of a collapse caused by pressure on a bronchus, but elucidation and investigation was prevented for some time by the patient's unwillingness to undergo endoscopy or surgery. This is a common obstacle to investigation of patients in these territories. The clinical suspicion that the lung lesion was a carcinoma could not be proved on this account.

The symptomatology would not have been helpful in making an ante-mortem diagnosis of teratoma. However, the radiological picture of cavitation at the periphery of the tumour is an unusual one and might have served as a clue that this was 
no ordinary tumour. As may be seen in the specimen, this cavity appears to coincide with a collection of fat in the teratoma.

Willis (1953) showed that the ovaries and testes are the commonest sites of teratomata. This fact was also observed by early workers who associated all extragenital teratomata with aberrant sex cells and even suggested that the mediastinal teratomata were arising in intrathoracic testis (Houghton, 1936). Schlumberger (1946) suggested that intrathoracic teratomata have their origin in faulty embryogenesis of the thymus. The genesis of teratomata, particularly intrathoracic teratomata, is far from being settled, and most of the theories on the subject are more or less speculative. Although there is a developmental relationship between the respiratory system and the thymus (Hamilton, Boyd and Mossman, 1962), the possibility of a thymic origin of these teratomata still lacks proof despite the fact that mediastinal and lung teratomata combined rank third in frequency of all teratoid tumours. In the light of recent advances in embryology, teratomata are likely to arise in areas of tissue that during early embryonic development escaped from the action of the primary organizer (Needham, 1942; Willis, 1951). Therefore, teratomata might arise in any tissue, not necessarily connected with the gonads or any of the sex-linked endocrine glands.

Benign teratomata are usually cystic, but some of the solid varieties are equally benign, and therefore the gross subdivision into these two types serves no useful purpose. It is also of no practical value to classify teratomata into monodermal, bidermal, and tridermal forms (Willis, 1951). However, well-developed pancreatic tissue, although infrequent in teratomata of other regions, is often seen in mediastinal and intrapulmonary teratoid tumours (Schlumberger, 1946; Collier et al., 1959). The case under review showed welldeveloped pancreatic tissue in the teratoma representing an entodermal derivative beside ectodermal components as well as areas of osteoid tissue representing mesodermal elements. Although the findings are in many respects similar to those reported by Collier et al. (1959), the 'nucleus' of adipose tissue and skin seems to be peculiar to this case. Various parts of the tumour examined in serial sections showed no evidence of malignant change. There were no other tumours, benign or malignant, in any of the other organs.

Six of the 15 cases of teratomata of the lungs listed in the Table were reported as malignant tumours. To complete the picture, a brief consideration of the conditions in which teratomata manifest malignancy will be included in this dis- $\overrightarrow{\vec{S}}$ cussion. Liebow (1952) drew attention to the fact that some intrapulmonary teratomata might repre- $\frac{\square}{0}$ sent metastatic lesions from an extrapulmonary $\frac{\bar{\sigma}}{\omega}$ source, such as those of the testes. Schiødt and $\mathbb{\nabla}$ Jensen (1960) made a point of excluding metastatic lesions when reporting their case of malignant ${ }^{\infty}$ teratoid tumour of the lung. It is not certain that $\vec{O}$

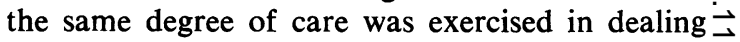
with all other cases of malignant teratomata $\omega_{\sigma}$ included in earlier case reports. Malignant changes $\vec{F}$ in teratomata are, in most cases, much more diffi- $\vec{x}$ cult to diagnose since malignancy might be $\vec{\omega}$ restricted to a localized area and only affect one $\omega$ of the tissue components of an otherwise benign- $N_{\infty}$ looking tumour. Although distant metastasis proves the malignant nature of the growth, neither failure of encapsulation nor local dissemination $\vec{z}$ are considered as malignant features in teratoid tumours. According to Willis (1951), three distinct $\overrightarrow{\mid}$ varieties of frankly malignant or locally spreading $\odot$ teratoid tumours are known. The first group in- + cludes those in which malignancy is shared by응 all tissue elements, a feature almost always seen in testicular teratomata. The second and commonest variety of malignant change in a teratoma generally affects only one component, such as the $\underset{\perp}{\mathscr{Q}}$ epidermis, and results in an invasive tumour which $\overrightarrow{\overrightarrow{0}}$ in all respects is similar to an epidermoid $\stackrel{\circ}{3}$ carcinoma. Serial sectioning and examination of $כ$ multiple areas in the tumour are essential in allo cases to prove or exclude malignancy. The third, peculiar variety of peritoneal dissemination is seen $\underline{0}$ in cases of ruptured intraperitoneal teratoid $\underset{x}{\stackrel{0}{\sim}}$ tumours. Multiple seeding by direct implantation $\dot{0}$ results, and all of the lesions might remain benign.

Teratoid tumours reported as showing malignant $\delta$ changes might, after surgical resection, result in an unexpectedly good prognosis. A follow-up of 0 the case of malignant teratoma reported by Barrett and Barnard (1945) showed that the patient was still alive and well in 1960-that is, 17 years after removal of the tumour (Barrett, 1963).

\section{SUMMARY}

The case is reported of a benign intrapulmonary teratoma in the right upper and middle lobes asso- 0 ciated with an atrial septal defect in a Chinese $\frac{\mathscr{D}}{\mathbb{C}}$ woman aged 59 years.

The rarity of intrapulmonary teratomata is $\square$ emphasized, and 14 other cases reported in the $\stackrel{D}{\circ}$

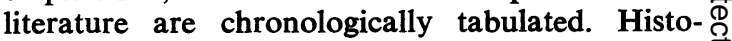
logically, the teratoma had ectodermal, meso- $\mathbb{\mathbb { Q }}$ dermal, and entodermal derivatives. Well-formed $\frac{\Omega}{\sigma}$ pancreatic tissue with islets of Langerhans, a 
feature recorded in other case reports, was seen in parts of the tumour. In the central portion of the tumour there was an unusual 'nucleus' of adipose tissue encased in well-developed skin and surrounded by heterogenous tissue.

A brief mention is made of the genesis of intrathoracic teratomata, and the criteria for diagnosing malignant changes are listed.

We wish to thank Professor K. Shanmugaratnam for his advice and review of the manuscript and Professor G. A. Ransome for permission to publish the case report. Many thanks are due to Mr. V. A. Nalpon for the photographs and to Mrs. Mary Low for the histological sections.

\section{REFERENCES}

Barrett, N. R. (1963). Personal communication.

and Barnard, W. G. (1945). Some unusual thoracic tumours (Case No. 5). Brit. J. Surg., 32, 447.

Black, H. R., and Black, S. O. (1918). Pulmonary teratoma-Report of a case with remarks on ovigenous tumors. Ann. Surg., 67, 73
Cloetta, A. (1861). Concerning the appearance of a dermoid cyst in the lung. Arch. path. Anat., $20,42$.

Collier, F. C., Dowling, E. A., Plott, D., and Schneider, H. (1959). Teratoma of the lung. Arch. Path., 68, 138 .

Hamilton, W. J., Boyd, J. D., and Mossman, H. W. (1962). Human Embryology, 3rd ed., p. 230. Heffer, Cambridge.

Houghton, J. D. (1936). Malignant teratoma of the mediastinum. Report of a case and review of 24 cases from the literature. Report of a case and
Amer. J. Path., 12, 349.

Inada, K., and Nakano, A. (1958). Structure and genesis of the mediastinal teratoma. Arch. Path., 66, 183.

Liebow, A. A. (1952). Atlas of Tumor Pathology, sect. 5, fasc. 17 : Tumors of the Lower Respiratory Tract, p. 139. Armed Forces Institute of Pathology, Washington, D.C.

Needham, J. (1942). Biochemistry and Morphogenesis, p. 231. University Press, Cambridge.

Ruland, L. (1956). Malignant teratoblastoma of the lung. Thoraxchirurgie, 4, 119.

Schiødt, T., and Jensen, K. G. (1960). Malignant teratoid tumour of the lung:? Malignant hamartoma. Thorax, 15, 120.

Schlumberger, H. G. (1946). Teratoma of the anterior mediastinum in the group of military age-A study of sixteen cases, and a review of theories of genesis. Arch. Path., 41, 398.

Spencer, H. (1962). Pathology of the Lung, p. 772. Pergamon Press, Oxford.

Willis, R. A. (1937). A further study of the structure of teratomata. J. Path. Bact., $45,49$.

(1951). Atlas of Tumor Pathology, sect. 3, fasc. 9: Teratomas, p. 11. Armed Forces Institute of Pathology, Washington, D.C. - (1953). Pathology of Tumours, 2nd ed., pp. 940-984. Butterworth, London. 